\title{
Ten years of asteroseismic modelling of pulsating B subdwarf stars: achievements, challenges, and prospects
}

\author{
S. Charpinet, ${ }^{1}$ G. Fontaine ${ }^{2}$ P. Brassard,${ }^{2}$ P. Chayer, ${ }^{3}$ E. M. Green, ${ }^{4}$ S. K. Randall ${ }^{5}$ \\ ${ }^{1}$ Observatoire Midi-Pyrénées, 14 Avenue E. Belin, 31400 Toulouse, France \\ ${ }^{2}$ Dépt. de Physique, Université de Montréal, Montréal, Québec, Canada, H3C 3J7 \\ 3 The Johns Hopkins University, Baltimore, Maryland 21218, USA \\ ${ }^{4}$ Steward Observatory, University of Arizona, Tucson Arizona 85721, USA \\ ${ }^{5}$ European Southern Observatory, Garching, Germany
}

\begin{abstract}
We present a short, non-exhaustive review of the major achievements and challenges resulting from a decade of modelling the pulsations in hot pulsating B subdwarf stars. We also briefly outline promising applications of sdB asteroseismology that will be explored in years to come, showing that this new domain of stellar astrophysics has many interesting ramifications and a strong potential for greatly improving our understanding of stellar structure and evolution.
\end{abstract}

\section{Introduction}

The year 2006 marks the tenth anniversary of the discovery of hot pulsating subdwarf B (sdB) stars. This discovery resulted from independent, but nearly simultaneous observational and theoretical efforts. The first pulsating sdB star, EC 14026-2647, was found by astronomers from the South African Astronomical Observatory (Kilkenny et al. 1997). In the meantime, Charpinet et al. (1996) had realized, after exploring the nonadiabatic pulsation properties of sdB stellar models, that physical conditions in the envelope of such stars were fulfilled to drive oscillation modes efficiently. This led, at that time, to the prediction that some sdB stars should be pulsating. Over the last decade, the joint development of theory and observation has led to significant breakthroughs in this field. We review some of them, as well as remaining difficulties, in the following sections. We also propose previews of what might be the future of sdB asteroseismology.

\section{Pulsations in Extreme Horizontal Branch Stars}

Hot B subdwarfs are believed to be the observed counterparts of the so-called Extreme Horizontal Branch (EHB) stars. In the HR diagram (or, equivalently, the $\log g-T_{\text {eff }}$ diagram; see Fig. 1), the EHB forms an extension to the blue of the classical Horizontal Branch. Stars associated with this phase of stellar evolution are thus expected to be evolved objects burning helium in their core. EHB stars are peculiar in that they must have been stripped down of almost all their $\mathrm{H}$-rich envelope during a previous evolutionary stage, leaving only a helium core with a mass usually expected in a narrow range centred around $0.48 M_{\odot}$ surrounded by an extremely thin residual envelope of mass lower than $\sim 0.02 M_{\odot}$. This configuration produces stars, clearly associated with the B subdwarfs, that remain hot ( $T_{\text {eff }}$ in the range $22000 \mathrm{~K}-$ $40000 \mathrm{~K})$ and compact $(\log g$ in the range $5.2-6.2)$ during their entire lifetime on the $\mathrm{EHB}$ ( $\sim 10^{8}$ years). In addition, such stars never ascend the AGB after core helium exhaustion and evolve instead as hot post-EHB stars - often identified to the observed subdwarf $O$ stars - before fading out as low-mass white dwarfs. Only a small fraction of the white dwarf population $(\sim 2 \%)$ is expected to have followed this path, however, as the vast majority 


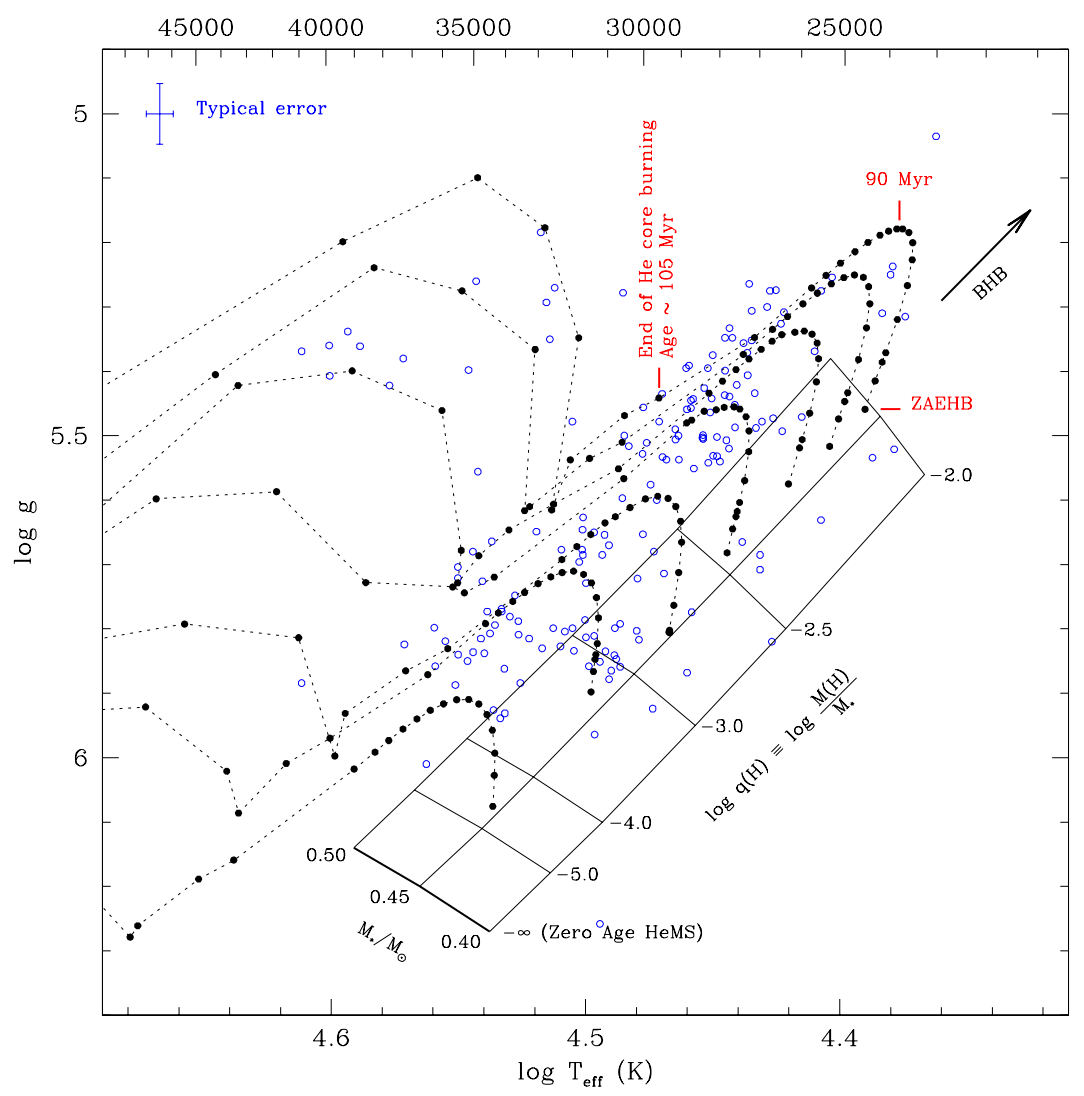

Figure 1: Illustration of the EHB region in the $\log g-T_{\text {eff }}$ plane. Representative EHB evolutionary tracks are shown (filled circles and dotted lines; from B. Dorman 1995, private comm.). Positions of EHB stars in this diagram are mainly determined by their total mass $M_{*}$ and the mass of their $\mathrm{H}$-rich envelope (the $\log q(H)$ parameter). The trends are illustrated with a grid of ZAEHB models (for $Z=0.02$ ) extending from the limit between the EHB and the Blue Horizontal Branch (BHB), at low $T_{\text {eff }}$, to the ZAHeMS, at high $T_{\text {eff }}$. A homogeneous sample of sdB stars with spectroscopic estimates of their atmospheric parameters is also shown (open circles).

of them is produced from post-AGB evolution. Major questions concerning the EHB phase of stellar evolution are still pending. One of the most intriguing is the process that lead to the formation of such stars. How EHB stars manage to lose all but a tiny fraction of their $\mathrm{H}$-rich envelope is, indeed, poorly understood. A number of competing scenarios have been proposed, from single star evolution to various binary evolution channels (e.g., mergers, common envelope evolution, stable and unstable Roche lobe overflow), but no clear solution has yet emerged.

Following the discovery of nonradial pulsations in B subdwarfs, the interest in EHB stars has been revived with the promise of improving our knowledge of this phase of stellar evolution 

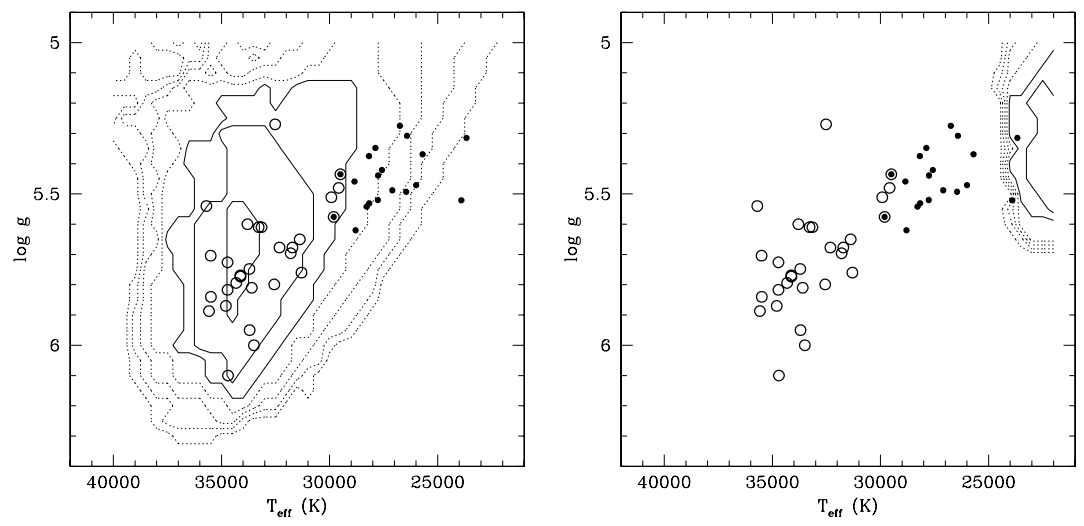

Figure 2: The EC 14026 pulsators (open circles) and Betsy stars (filled circles) with their predicted instability strips in the $\log g-T_{\text {eff }}$ plane. In the left panel, contours indicate the number of excited $\ell=0 \mathrm{p}$ modes (with a maximum near $T_{\text {eff }} \sim 34000 \mathrm{~K}$ and $\log g \sim 5.7$ ) in the nonadiabatic models. In the right panel, contours show the number of excited $\ell=4 \mathrm{~g}$ modes. The highest contours corresponding to regions of highest efficiency for the driving mechanism are drawn as solid-lines.

through the use of asteroseismology. Two classes of sdB pulsators are presently known (see also the review by Kilkenny 2007). The V361 Hya stars (or EC 14026 stars from the name of the prototype) - the first that were discovered in 1997 - form a group of rapid pulsators showing luminosity variations on a timescale of a few minutes (from 80 to 600 seconds, typically). They tend to cluster around values of $T_{\text {eff }} \sim 33000 \mathrm{~K}$ and $\log g \sim 5.8$ in the $\log g-T_{\text {eff }}$ plane, but are found within a relatively wide range of parameters ( $T_{\text {eff }} \sim 33000-$ $36000 \mathrm{~K}$ and $\log g \sim 5.2-6.2$; see Fig. 2). Comparisons with models immediately indicate that the involved pulsations are radial and nonradial, low-order and low-degree sound waves (or p modes). The second class of sdB pulsators, the PG $1716+426$ stars (from the prototype, but often referred to as the "Betsy stars"), was discovered in 2003 (Green et al. 2003). These stars show slow luminosity variations on a timescale of hours (from 2000 to 9000 seconds) which are associated with relatively high-order gravity modes (or g modes). They are cooler than the EC 14026 stars, having $T_{\text {eff }}$ and $\log g$ in the ranges $23000-30000 \mathrm{~K}$ and $5.2-$ 5.6, respectively (see Fig. 2). Interestingly, two stars (HS 0702+6043 and BAL 090100001) are known to show both $\mathrm{p}$ - and g-mode pulsations, thus belonging to the two classes of pulsators. Hence, the blue edge of the Betsy star instability strip overlaps with the red-edge of the EC 14026 instability region.

\section{Understanding the pulsations in hot B subdwarf stars}

The nature of the driving mechanism for the EC 14026 stars was identified very early (Charpinet et al. 1996). It relies on two essential components. The first is the presence of heavy metals, especially from the iron-group, through their impact on the gas opacity. In EHB stellar models, heavy metals produce an opacity bump - the so-called Z-bump - that is ideally located in the envelope, thus producing an effective pulsation driving mechanism through a $\kappa$-effect. This driving, however, is not strong enough in standard EHB models assuming solar $(Z \sim 0.02)$ metallicity and needs to be enhanced to destabilize modes. This is where the second essential component, microscopic diffusion, comes into play. Subdwarf B stars are notorious for having 
chemically peculiar atmospheres. Helium is usually highly depleted by large amounts while other elements show complex, non-solar abundance patterns. These are commonly attributed to the competing action of radiative levitation, gravitational settling, and possibly weak stellar winds. Detailed calculations show that, indeed, the very stable radiative envelopes of sdB stars offer auspicious conditions for microscopic diffusion to modify their internal chemical composition. In particular, elements from the iron-group tend to accumulate in the envelope, thanks to radiative levitation. This results in a strong amplification of the $Z$-bump and of the associated $\kappa$-effect, leading to the excitation of pulsation modes. It was later shown by Fontaine et al. (2003) that this mechanism is also responsible for the pulsations seen in Betsy stars. Diffusion is therefore a fundamental ingredient that must be taken into account if one wants to understand and exploit the pulsations in sdB stars. Our current EHB models (referred to as the "second generation" models) implement the nonuniform abundance profile of iron (the main contributor to the Z-bump) derived from detailed diffusion calculations assuming equilibrium between radiative levitation and gravitational settling.

Comparisons between the theoretical and observed properties of $\mathrm{sdB}$ pulsators using these second generation models are numerous (see, e.g., Charpinet et al. 2006a). One of particular interest concerns the theoretical instability strips which are illustrated in Fig. 2. In this figure, the contours provide a view of the efficiency of the driving mechanism as a function of $\log g$ and $T_{\text {eff }}$ for both the rapid p-mode pulsators (left panel) and the g-mode pulsators (right panel). Clearly the correspondence between observations and models is excellent in the case of the EC 14026 pulsators, especially as the driven modes have periods identical to those actually seen in these stars. Nonetheless, difficulties remain considering that the theoretical p-mode instability strip is wider than observed and because pulsators and non-pulsators coexist (with a ratio $\sim 1$ over 10) in the same region of the $\log g-T_{\text {eff }}$ plane. Some of these issues, however, will likely be solved with further refinements in the modelling of the diffusion processes in sdB stars (see, e.g., Fontaine et al. 2006). The situation for the long-period Betsy stars has been more intriguing. In our second generation models, g-mode instabilities are indeed found for relatively high degree $(\ell>3)$, high-order $\mathrm{g}$ modes with periods in the range of those observed. The theoretical blue-edge of the g-mode instability region is, however, much too cool compared to the atmospheric parameters derived for the known Betsy stars from spectroscopy. This discrepancy $(\sim 5000 \mathrm{~K}$ for $\ell=3-4$ modes, as the blue edge depends, in fact, on the $\ell$ index of the modes) has been a major puzzle over the last 3 years. A significant breakthrough toward a solution to this problem was made recently by Jeffery \& Saio (2006; see also these proceedings). While exploring the effects of nickel abundance enhancements on the Z-bump and the driving mechanism, these authors found that adding $\mathrm{Ni}$ and using opacities from the Opacity Project (as opposed to OPAL) result in blue-shifting the g-mode instability strip by $\sim 5000 \mathrm{~K}$. Hence, a reinvestigation of the driving mechanism with models including these new elements, and in particular nonuniform profiles of $\mathrm{Ni}$ predicted by diffusion calculations, is needed.

\section{Asteroseismology of subdwarf B stars}

The most recent activities on sdB pulsators have been attempts to model individual stars in detail. The aim is to fully and accurately reproduce the observed pulsation period spectra and to isolate the model that corresponds most closely to the star being studied, hence constraining the stellar structure of EHB stars from asteroseismology. Both the EC 14026 and Betsy stars present a high potential for asteroseismology, but the EC 14026 pulsators have received the highest attention in this area, so far (however, see Randall et al. 2006 for a first tentative of asteroseismic analysis of a Betsy star).

In the recent years, we have set up a new global approach to the problem of asteroseismology of EC 14026 pulsators. Our technique - a global optimization procedure - allows us to exhaustively and efficiently explore the vast model parameter space in order to isolate the 


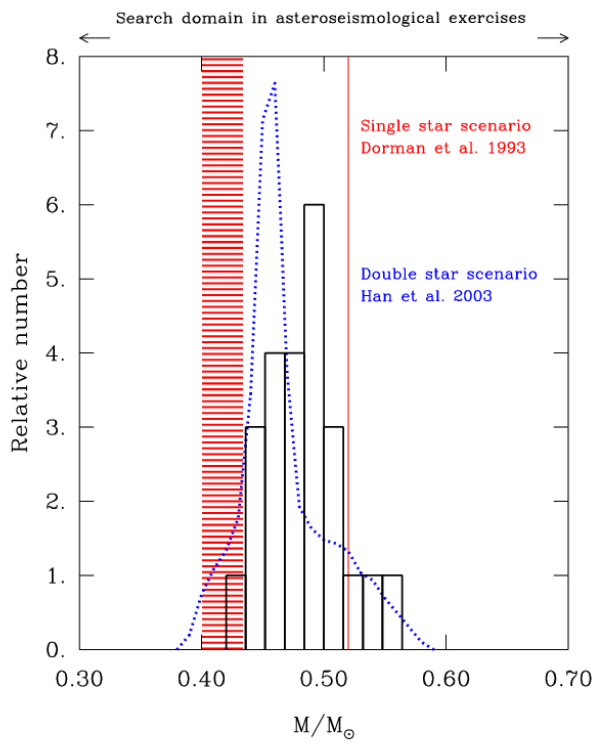

Figure 3: The preliminary empirical mass distribution of sdB stars as derived from asteroseismology of nine EC 14026 stars (histogram). In comparison, two theoretical distributions are also shown (vertical lines: the single star evolution scenario. Dotted curve: the double star evolution scenario).

model that can best-match the period spectrum of the EC 14026 pulsator under study. Developed mainly in the context of interpreting white light fast-photometric data for which no a priori information on the mode identification exists, our procedure is a "double-optimization" scheme that simultaneously searches for the optimal combination of observed and computed periods (for a model with given parameters) and for the optimal set of model parameters. This method leads objectively to the best match of the observed periods, providing estimates of the structural parameters of the star and a complete mode identification (i.e., the $\ell$ and $k$ indices) of the observed periods. Currently, six EC 14026 pulsators have been fully analysed with this method. These are PG 0014+067, PG 1047+003, PG 1219+534, Feige 48, EC 20117-4014, and PG 1325+101 (see Charpinet et al. 2006b and reference therein). In addition 3 other EC 14026 stars have been studied in a preliminary form. In all cases, a best-fit model solution completely consistent with the atmospheric parameters of the star estimated from spectroscopy has been found. In all cases, all the observed periods are matched to driven modes, according to nonadiabatic calculations. Typically, the observed and computed periods are matched with an average dispersion of $\langle\Delta P / P\rangle \sim 0.5 \%$ or, on an absolute scale, $<\Delta P>\sim 0.5 \mathrm{~s}$ or $\langle\Delta \nu>\sim 40 \mu \mathrm{Hz}$ (as a comparison, typical large spacings for acoustic modes are $\sim 1000-1600 \mu \mathrm{Hz}$ in these stars). Acoustic modes of degree $\ell=0-4$ are involved (and usually required by the observed mode density). Clearly, improvements at the level of the stellar models are needed, especially as the accuracy at which the frequencies (or periods) are determined with our current observations $(0.5-3 \mu \mathrm{Hz}$ is the typical resolution achieved) is still one order of magnitude better than the mean dispersion of the best-fit models. This leaves significant room for future refinements in the description of EHB stellar structures. Note also that tests of the seismic models are possible with multicolour photometry leading to the independent identification of the $\ell$ index of some of the observed modes. These can then be compared with the mode identification derived from the asteroseismic analysis. 


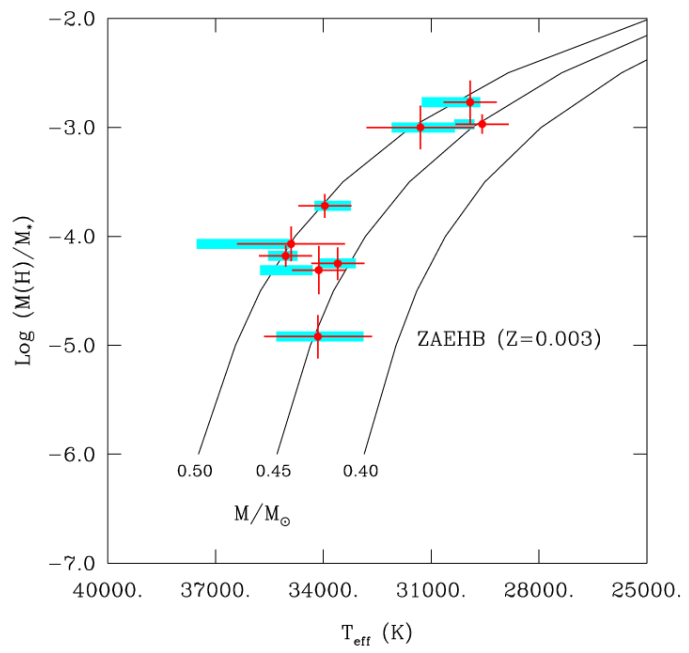

Figure 4: Expected (solid curves) and observed (dots with error bars) correlations between the mass of the $\mathrm{H}$ envelope, the effective temperature, and the total mass of the nine EC 14026 pulsators analysed. This shows a nice test of stellar evolution theory and illustrates the strong internal consistency that exists between the derived parameters (thick horizontal bands indicate the mass derived from asteroseismology).

\section{The future of B subdwarf asteroseismology}

A preview of some of the most interesting prospects of sdB asteroseismology is given in Fig. 3 and Fig. 4 (see figure captions for details). Objectives are to help identify the evolutionary channels that lead to the formation of EHB stars, for instance by comparing mass distributions derived from asteroseismology to those expected from different formation scenarios (Fig. 3).

Moreover, asteroseismology of $\mathrm{sdB}$ stars now shows a strong potential for accurately testing EHB structure and stellar evolution (Fig. 4). In both cases, the number of stars analysed so far is insufficient to draw firm conclusions on these topics, but it will become possible with improved statistics.

\section{References}

Charpinet S., Fontaine G., Brassard P., Dorman B., 1996, ApJ, 471, L103

Charpinet S., Fontaine G., Brassard P., Chayer P., Green E. M., 2006a, Baltic Astron., 15, 305

Charpinet S., Silvotti R., Bonanno A., et al., 2006b, A\&A, 459, 565

Fontaine G., Brassard P., Charpinet S., et al., 2003, ApJ, 597, 518

Fontaine G., Green E. M., Chayer P., et al., 2006, Baltic Astron., 15, 211

Green E. M., Fontaine G., Reed M. D., et al. 2003, ApJ, 583, L31

Han Z., Podsiadlowski P., Maxted P. F. L., Marsh T. R., 2003, MNRAS, 341, 669

Jeffery C. S., Saio H., 2006, MNRAS, 372, L48

Kilkenny D., 2007, Comm. Asteroseis., 150, 234

Kilkenny D., Koen C., O'Donoghue D., Stobie R. S., 1997, MNRAS, 285, 640

Randall S. K., Green E. M., Fontaine G., et al., 2006, ApJ, 645, 1464 